\title{
A Summary of Recent Advances in Ocular Inserts and Implants
}

\section{Jervis LP*}

Pharmaceutical Sciences, Fairleigh Dickinson University, Teaneck, NJ 07666, USA

\begin{abstract}
With the physiological and anatomical constraints put forth by the eye it is a significant challenge to the pharmaceutical scientists to target drugs to the posterior segment of the eye. Severe ocular complications such as glaucoma, uveitis, cytomegalovirus retinitis, cataracts, age related macular degeneration, diabetic retinopathy, and retinitis pigmentosa pose urgency for the treatment of ocular diseases. Hence novel drug delivery strategies and formulations are to be developed and explored to overcome and treat these posterior ocular disorders. At present the topical and intravitreal routes are widely used to deliver therapeutic entities to retinal tissue. Various controlled delivery systems, such as ocular inserts and implants are in the progress of development and may improve drug delivery drastically in the years to come. In this review, we discuss the recent developments in ocular delivery using ocular implants and inserts.
\end{abstract}

Keywords: Ocular; Implants; Inserts; Intravitreal; Eye

Abbreviation: BAB: Blood-Aqueous Barrier; BRB: BloodRetinal Barriers; IOP: Intraocular Pressure; PLA: Polylactic Acid; PGA: Polyglycolic Acid; PLGA: Polylactic-co-Glycolic Acid; PCL: Polycaprolactones; PVA: Polyvinyl Alcohol; EVA: Ethylene Vinyl Acetate; PCF: Polysulfone Capillary Fiber; Gd-DTPA: GadoliniumPentetic Acid; TA: Triamcinolone Acetonide; RC: Retina-Choroid; DME: Diabetic Macular Edema; FA: Flucinolone Acetonide; GCV: Ganciclovir; CMV: Cytomegalovirus; PLGA: Polylactic-Glycolic acid; BRVO: Branch Retinal Vein Occlusion; CRVO: Central Retinal Vein Occlusion; DEX: Dexamethasone

\section{Introduction}

Targeting drugs to the back of the eye is a highly challenging task. Drug delivery to posterior segments of the eye through the existing topical route still remains challenging, it is established that typically, only $1 \%$ or less of a topically instilled dose was delivered into the anterior segments, and a negligible amount to the posterior segment $[1,2]$. Oral or systemic administration of therapeutic entities is not effective because of Blood-Aqueous Barriers (BAB) and Blood-Retinal Barriers (BRB), limiting the passage of drugs into the eye from the systemic circulation $[3,4]$. Intravenous administration is effective to maintain the drug concentrations in the posterior tissues relatively at high doses but pose adverse effects and systemic toxicity. Currently, intravitreal injection (i.e., direct injection of a drug into the vitreous body) is reported to be the most promising and unique method of delivering a drug to ocular posterior segments but is too invasive technique and is associated with complexities like retinal detachment, cataract, endophthalmitis and increased intraocular pressure [5-7]. Subconjunctival injection, administration of drug into the region between the conjunctiva and the sclera (subconjunctival space), is reported for back-of-the eye delivery through the trans-scleral route. The subconjunctivally injected drugs have direct contact with the sclera, so that the trans-scleral transport of drugs may be quite higher. Moreover, subconjunctival injection has the ability to circumvent the conjunctival absorption, which is considered to be a barrier in terms of permeability. Consequently, periocular and intravitreal routes of administration serves as viable platforms for the delivery of drugs to posterior tissues [8,9]. Depending upon the ophthalmic disease state, complexity and origin delivery systems such as intraocular implants, intravitreal injections $[10,11]$. Even though the tight cellular junctions and various barriers of eye restrict the diffusion and transport of drugs, administration of therapeutic agents through various novel ophthalmic inserts and implants is of interest to various researchers. Ocular implants and inserts are such novel strategies that are designed to circumvent these ocular barriers and facilitate the drugs to the posterior section of the eye [12-14]. In the current review the recent developments about the ocular inserts and implants are discussed in detail.

\section{Ocular Inserts}

Ocular inserts are sterile, multi-layered, drug-impregnated devices placed into the cul-de-sac or conjunctival sac of the eye for the prolonged release of medication. The inserts are classified based on their physicochemical properties as insoluble, soluble, or bioerodible [15]. Soluble inserts, also are erodible, for example monolytic polymeric devices that undergo gradual dissolution while releasing the drug, and do not need removal. Insoluble inserts are further classified into Reservoir and matrix systems and they can usually deliver drugs in controlled, predetermined rate, but need removal from the eye. Collagen shields, occfit, mini disc ocular therapeutic systems are the ocular inserts later developed [16-18]. In one study Hassan et al. formulated the brimonidine sodium alginate ocular inserts and observed the sustained drug release rate and Intraocular Pressure (IOP) reduction in the rabbit eyes. The release of the drug (in vitro) was for $6 \mathrm{~h}$ and the IOP reduction was $40 \%$. The AUC values observed were $\sim 4.5$-folds higher than that of the instilled topical eye drops [19]. Amar et al. investigated the release of Betaxolol hydrochloride from the ocular inserts and the drug release from in vitro studies was $98.76 \%$ at the end of $12 \mathrm{~h} \mathrm{[20].}$ Alan et al. patented biodegradable controlled release ocular insert of chloramphenicol sodium monosuccinate impregnated with Polylactic Acid (PLA) as treatment for infectious bovine kerato-conjunctivitis. The ophthalmic insert will be retained in third eyelid of mammals and would be able to deliver drug for seven consecutive days [21,22].

*Corresponding author: Lynda Paul Jervis, Pharmaceutical Sciences, Fairleigh Dickinson University, 1000 River Road, Teaneck, NJ, USA, Tel: 609-738-7000; Fax: 973 443-8795; E-mail: Iyndajervis1968@gmail.com

Received November 30, 2016; Accepted December 16, 2016; Published January 02, 2017

Citation: Jervis LP (2017) A Summary of Recent Advances in Ocular Inserts and Implants. J Bioequiv Availab 9: 320-323. doi: 10.4172/jbb.1000318

Copyright: (c) 2017 Jervis LP. This is an open-access article distributed under the terms of the Creative Commons Attribution License, which permits unrestricted use, distribution, and reproduction in any medium, provided the original author and source are credited. 


\section{Implants}

Recent technological advances in the field of biomedical engineering and ocular surgery fortified the insight of design and development of sustained-release drug delivery implants for the treatment of various clinical ophthalmic complications $[23,24]$. Sustained release ocular implants are the drug delivery devices for sustained release of molecules from either biodegradable or non-biodegradable polymeric matrices over several months to years. The foremost intraocular implants were developed in order to achieve controlled and sustained drug delivery to treat long term ophthalmic disorders. These devices can be implanted in subconjuctival, episcleral, intravitreal, intracameral regions. Biodegradable solid implants are fabricated using Polylactic Acid (PLA), Polyglycolic Acid (PGA), and Polylactic-co-Glycolic Acid (PLGA) Polycaprolactones (PCL) polyanhydrides which does not need post treatment surgical removal unlike non-biodegradable implants, but can cause erratic drug release profiles [25]. Kochinke et al. patented the fabrication of dexamethasone monolithic ocular implant using polyester of lactic and glycolic acid with hydroxypropyl methyl cellulose which could deliver drug for the period of 3 days. This implant can be inserted into various sites of the eye depending up on the ailment and condition to be treated [26,27]. Non-biodegradable implants are made up of polyvinyl alcohol (PVA)-Ethylene Vinyl Acetate (EVA), Polysulfone Capillary Fiber (PCF). Rahimy et al. conducted studies to investigate PCF as drug delivery device for intraocular applications. Carboxyfluorescein (CF) was used as the model drug for these studies and the subsequent release kinetics of CF from the PCF device was monitored in vivo in the rabbit's eye. PCF dye device was implanted in the vitreous cavity, and fluorophotometry from the retina to the anterior chamber was performed at various times up to 45 days to quantify fluorescein level. At the conclusion of the study, eyes were enucleated and examined for histopathology. The time-course study showed fluorescein level for up to 45 days in the vitreous and further histological examination of the eyes implanted with PCF or PCF-dye device showed no sign of ocular toxicity. Overall, these results may imply that the PCF device is biocompatible and may be useful for the extended release of drugs in the posterior segment of the eye [28-30]. Kim et al. attempted to Deliver Gadolinium-Pentetic Acid (Gd-DTPA) to the posterior segment by episcleral sustained release implant and the release rate of the episcleral implant was compared with intravitreal implant (in vivo). Episcleral implants delivered $2.7 \mu \mathrm{g}$ in to the vitreous cavity comprising only $0.12 \%$ of the drug in the implant and there were no significant amounts of the drug in the posterior chamber. Intravitreal implants delivered the drug in to the vitreous humour and posterior segments of the eye. The concentration of the drug in the vitreous was 30 times higher (ex vivo) when compared to episcleral implant. Author hypothesized that three-dimensional MRI and the data would be useful to study the ocular disposition mechanisms in the eye [31]. In other study Kim YM et.al delivered the triamcinolone acetonide into posterior segment of the eye using the intrascleral implant. The implant was made of poly (D,L-lactide) comprising $6.4 \mathrm{mg}$ of Triamcinolone Acetonide (TA). Sustained release of triamcinolone acetonide for 90 days was observed in the in vitro studies and a significant level of TA in aqueous humor until 4 weeks was detected and in retina-choroid until 8 weeks after implantation, but in the vitreous cavity TA was found over 12 weeks. There were no signs of retinal detachment or toxicity in the in vivo studies [32]. Gwon and Meadows patented the subconjunctival implants of pilocarpine which could significantly deliver and control the release of drugs into the ocular posterior segment. The implants are made of ethylene vinyl acetate polymer which controls the release of drug; amount of drug disposed into conjunctiva can be determined by fluorescent tracer embedded in the subconjunctival implant [33].
Okaba et al. prepared and evaluated biodegradable scleral implant for the sustained release of steroid betamethasone phosphate to the posterior segments of the eye (in vitro). The implant with dimensions of $0.5 \mathrm{~mm}$ thick and $4 \mathrm{~mm}$ in diameter made up of poly (DL-lactide) was inserted in to scleral pocket of the rabbit's eye. The drug levels in the posterior tissues like vitreous and Retina-Choroid (RC) was maintained constant for 8 weeks. The drug concentration in the RC was significantly greater than in the vitreous humor. Drug levels in the aqueous humor were below limit of detection. The implant exerted good compatibility in eye and there was no significant toxicity to the retina during the experimental studies [34,35]. Michelson and Nozik fabricated implantable osmotic mini pump device, which was inserted subcutaneously in the ear region of a rabbit model of endophthalmitis. The device had connective tubing directly infusing into the vitreous cavity through a pars plana incision and maintained a calculated dose of the antibiotic gentamicin $(0.01 \mathrm{mg} / \mathrm{h})$ over 4 days [36]. Silvia et.al formulated dexamethasone intravitreal implants made up of polymer poly( $\varepsilon$-caprolactone) which is suitable for the long term sustained release drug delivery to the vitreous humor. Characteristics of the poly ( $\varepsilon$-caprolactone) device, feasibility and intravitreal release of dexamethasone were extensively studied in this experiment. In vitro release of dexamethasone was determined and interaction between the drug and the polymer was evaluated by the differential scanning colorimetry. Poly( $\varepsilon$-caprolactone) device provided the sustained release of dexamethasone since it releases $25 \%$ of the drug loaded in 21 weeks. There were no significant changes during the experimental studies with morphology, toxicity, and other biological characteristics $[37,38]$. Carcaboso et al. attempted to deliver topotecan to the posterior segment of the eye for the treatment of intraocular retinoblastoma. Episcleral Implant was developed to control and sustain the delivery of topotecan in to the posterior segment. Implants released $30 \%$ to $50 \%$ of the drug within 48 hours and $45 \%$ to $70 \%$ by $10^{\text {th }}$ day (in vitro). In vivo, topotecan lactone was highly accumulated in ocular tissues such as (sclera, choroid, retina) over 48 hours with all the formulations studied. Low vitreous topotecan lactone levels were detected with high drug load implants [39]. Ganciclovir loaded biodegradable donut shaped minitablet was developed by Choonara et al. for the treatment of human cytomegalovirus retinitis. Specialised tablet tooling equipment was used to manufacture the device composed of polylactic-co-glycolic acid. Device was implanted through parsplana/peripheral retina of rabbits and the left eyes were used as control. The minitablet was well tolerated up to 72 days in super temporal quadrant of the eye. The device exhibited the control release of ganciclovir at the constant rate of $2.02 \mu \mathrm{g} / \mathrm{h}$ throughout the experimental studies [40].

\section{Current Marketed Products}

\section{Iluvien"}

Iluvien is sustained release flucinolone acetonide formulation undergone phase 3 clinical trials for treatment of Diabetic Macular Edema (DME). It's an injectable, non-erodible intravitreal implant for the treatment of DME. Iluvien is designed for sustained release of the formulation for over three years. Implant is injected into back-of-the eye using $25 \mathrm{G}$ needle creating self-healing hole which is very similar to intravitreal injection. Currently, the only FDA approved method for treating DME involves laser photocoagulation therapy which can leave irreversible blind spot.

\section{Retisert}

Retisert is (Flucinolone Acetonide (FA) intravitreal implant) for treatment of chronic, non-infectious posterior uveitis. Retisert 
is surgically implanted into vitreous humor by $3-4 \mathrm{~mm}$ incision containing $0.59 \mathrm{mg}$ of flucinolone acetonide which delivers the medicament up to 2.5 years. Retisert implant is composed of a central core consisting of FA compressed into a $1.5 \mathrm{~mm}$ diameter. Each FA tablet is enclosed in a silicone elastomer cup containing a release orifice. A semi-permeable layer of PVA coats the tablet inside the cup reservoir near the release orifice, creating a membrane between the tablet and the orifice that serves as an additional barrier for drug release from the cup. A suture tab, made from PVA film, is attached to the silicone cup using silicone adhesive. The silicone adhesive and the elastomer material are impermeable to FA, while PVA is permeable to diffusion of the drug. By varying the size of the elastomer cup's release orifice and the permeability of the PVA layer between the tablet and the orifice, the rate of drug release from the implant can be controlled. Release of FA from the cup reservoir occurs as water from the exterior of the implant penetrates into it and dissolves some of the drug. The dissolved drug substance then diffuses across the release orifice through the semipermeable layer of PVA into the medium.

\section{Durasert $^{\mathrm{Tm}}$}

Durasert" technology system (pSivida Corp., Watertown, MA, USA) uses a drug core with one or more surrounding polymer layers, and delivers drugs for predetermined periods of time ranging from days to years. The drug release is controlled by permeability of the polymer layers [41,42].

\section{Vitrasert}

Using the Durasert" system, an antiviral drug, ganciclovir (GCV)loaded intravitreal implant (Vitrasert, Bausch and Lomb Inc., Rochester, NY, USA) has been developed for the treatment of AIDS related cytomegalovirus (CMV) retinitis, that avoids systemic side effects and minimizing frequent intravitreal injections. This approach can significantly delay progression of CMV when compared with conventional ganciclovir intravenous treatment. The implant is made of EVA and PVA, surgically placed in the posterior segment of the eye releases GCV locally to the site of infection by passive diffusion through a small opening in EVA at the base of the device for 6-8 months.

\section{Ozurdex}

Ozurdex" (Allergan, Inc., Irvine, CA, U.S.) is an intravitreal implant containing $0.7 \mathrm{mg}$ of Dexamethasone (corticosteroid) composed of Polylactic-Glycolic acid (PLGA) (length: $6.5 \mathrm{~mm}$, diameter: $0.45 \mathrm{~mm}$ ) approved by FDA in June, 2009 for the treatment of chronic uveitis and macular edema following Branch Retinal Vein Occlusion (BRVO) and Central Retinal Vein Occlusion (CRVO). Ozurdex is administered by specially designed injector with a 22 -gauge needle into vitreous cavity. The most adverse effects include increase of intraocular pressure (25\%), conjunctival hemorrhage (22\%), eye pain $(8 \%)$, conjunctival hyperemia (7\%), cataract (5\%), vitreous detachment (2\%), and headache (4\%) [43].

\section{I-Vation}

SurModics, Inc. (Eden Prairie, MN, U.S.) has an I-vation"' drug delivery platform technology for the delivery of triamcinolone acetonide (TA) into the vitreous humor. I-vation" is the intravitral implant with titanium helical coil (length; $0.5 \mathrm{~mm}$, width; $0.21 \mathrm{~mm}$ ) coated with TA $(925 \mu \mathrm{g})$ and non-biodegradable polymers namely poly (methyl methacrylate) and ethylene vinylacetate. It is predicted that this implant will have an in vivo sustained delivery for a minimum of two years. At present I-vation is in the 1st phase of clinical trials $[43,44]$.

\section{Cortiject}

Cortiject (Novagali Pharma S.A.) is a preservative-free emulsion composed of oily carrier and phospholipid as surfactant, encapsulating corticosteroid prodrug with activated tissue targeting mechanism. Released dexamethasone (DEX) palmitate is de-esterified by a retinaspecific esterase and activated to be DEX. A single intravitreal injection provides sustained release for 6-9 months. Cortiject is under the phase I studies and need to be investigated [45].

\section{Conclusion}

It's evident that drug delivery to the posterior segments of eye presents significant and considerable confrontations. As, systemic administration is not effective prior to high drug doses, toxicity, blood retinal and aqueous barriers novel technologies needs to be primarily designed and to be developed to provide sustained action, enhance bioavailability, improved patient safety and minimal adverse effects. Ocular inserts and implants are to be developed to accommodate the increasing number of patients requiring long term progressing treatments. Eventually, multidisciplinary integration of delivery technologies to optimize drug bioavailability is needed. Developments in fields of biomedical engineering, nanotechnology and non-invasive drug delivery could open up the possibilities for drug delivery to the ocular posterior segments in the near future.

\section{References}

1. Lee TWY, Robinson JR (2001) Drug delivery to the posterior segment of the eye. I: Some insights on the penetration pathways after subconjunctival injection. J Ocul Pharmacol Ther 17: 565-572.

2. Adelli GR, Balguri SP, Punyamurthula N, Bhagav P, Majumdar S (2014) Development and evaluation of prolonged release topical indomethacin formulations for ocular inflammation. Invest Ophthalmol Vis Sci 55: 463.

3. Cunha-Vaz J (1979) The blood-ocular barriers. Surv Ophthalmol 23: 279-296.

4. Balguri SP, Adelli GR, Majumdar S (2016) Topical ophthalmic lipid nanoparticle formulations (SLN, NLC) of indomethacin for delivery to the posterior segment ocular tissues. Eur J Pharm Biopharm 109: 224-235.

5. Macha S, Mitra AK (2002) Ocular disposition of ganciclovir and its monoester prodrugs following intravitreal administration using microdialysis. Drug Metab Dispos 30: 670-675.

6. Maurice DM (1976) Injection of drugs into the vitreous body. In: Symposium on Ocular Therapy, Leopold I, Burns R, John Wiley \& Sons Inc., London, pp: 59-72.

7. Adelli GR, Balguri SP, Majumdar S (2015) Effect of cyclodextrins on morphology and barrier characteristics of isolated rabbit corneas. AAPS Pharm Sci Tech 16: $1220-1226$.

8. Duvvuri S, Majumdar S, Mitra AK (2003) Drug delivery to the retina: challenges and opportunities. Expert Opin Biol Ther 3: 45-56.

9. Saha P, Uchiyama T, Kim KJ, Lee VH (1996) Permeability characteristics of primary cultured rabbit conjunctival epithelial cells to low molecular weight drugs. Curr Eye Res 15: 1170-1174.

10. Hsu J (2007) Drug delivery methods for posterior segment disease. Curr Opin Ophthalmol 18: 235-239.

11. Balguri SP, Adelli G, Bhagav P, Repka MA, Majumdar S (2015) Development of nano structured lipid carriers of ciprofloxacin for ocular delivery: Characterization, in vivo distribution and effect of PEGylation. Invest Ophthalmol Vis Sci 56: 2269.

12. Del Amo EM, Urtti A (2008) Current and future ophthalmic drug delivery systems. A shift to the posterior segment. Drug Discov Today 13: 135-143.

13. Kaur IP, Kanwar M (2002) Ocular preparations: the formulation approach. Drug Dev Ind Pharm 28: 473-493.

14. Adelli GR, Hingorani T, Punyamurthula N, Balguri SP, Majumdar S (2015) Evaluation of topical hesperetin matrix film for back-of-the-eye delivery. Eur $\mathrm{J}$ Pharm Biopharm 92: 74-82. 
15. Kumari A, Sharma PK, Garg VK, Garg G (2010) Ocular inserts - Advancement in therapy of eye diseases. J Adv Pharm Technol Res 1: 291-296.

16. Kumar KPS, Bhowmik D, Duraivel S, Harish G, Kumar P (2013) Ocular Inserts: A Novel Controlled Drug Delivery System. J Pharm Innov 1: 1-16.

17. Saettone FM, Salminen L (1995) Ocular inserts for topical delivery. Turkey J Pharm Sci 16: 95-106.

18. Köllmer M, Popescu C, Manda P, Zhou L, Gemeinhart RA (2013) Stability of benzocaine formulated in commercial oral disintegrating tablet platforms. AAPS Pharm Sci Tech 14: 1333-1340.

19. Aburahma MH, Mahmoud AA (2011) Biodegradable Ocular Inserts for Sustained Delivery of Brimonidine Tartarate: Preparation and In vitro/In vivo Evaluation. AAPS Pharm Sci Tech 12: 1335-1337.

20. Amar A, Ashish K, Ajaykumar P, Anand J (2012) Formulation and evaluation of controlled release ocular inserts of betaxolol hydrochloride IOSR J Pharm 2: 34-38.

21. Alan HBII, Theodorakis MC (1984) Biodegradable ocular insert for controlled delivery of ophthalmic medication. Google Patents.

22. Yang Y, Manda P, Pavurala N, Khan MA, Krishnaiah YS (2015) Development and validation of in vitro-in vivo correlation (IVIVC) for estradiol transdermal drug delivery systems. J Control Release 210: 58-66.

23. Langer R (1990) New methods of drug delivery. Science 249: 1527-1533.

24. Manda P, Angamuthu M, Hiremath SR, Raman V, Murthy SN (2014) lontophoretic drug delivery for the treatment of scars. J Pharm Sci 103: 16381642.

25. Miller RA, Brady JM, Cutright DE (1977) Degradation rates of oral resorbable implants (polylactates and polyglycolates): rate modification with changes in PLA/PGA copolymer ratios. J Biomed Mater Res 11: 711-719.

26. Kochinke F, Wong $\vee(1999)$ Formulation for controlled release of drugs by combining hydrophilic and hydrophobic agents. Google Patents.

27. Loria MJ, White SW, Robbins SA, Salmeto AL, Hymel KA, et al. (2013) Brainderived neurotrophic factor response in vulnerable and resilient genetic lines in the chick anxiety-depression model. Behav Brain Res 245: 29-33.

28. Rahimy MH, Peyman GA, Chin SY, Golshani R, Aras C, et al. (1994) Polysulfone capillary fiber for intraocular drug delivery: in vitro and in vivo evaluations. J Drug Target 2: 289-298.

29. Smith TJ, Pearson PA, Blandford DL, Brown JD, Goins KA, et al. (1992) Intravitreal sustained-release ganciclovir. Arch Ophthalmol 110: 255-258.

30. Manda P, Hargett JK, Kiran Vaka SR, Repka MA, Narasimha Murthy S (2011) Delivery of cefotaxime to the brain via intranasal administration. Drug Dev Indust Pharm 37: 1306-1310.
31. Kim H, Robinson MR, Lizak MJ, Tansey G, Lutz RJ, et al. (2004) Controlled drug release from an ocular implant: an evaluation using dynamic three-dimensional magnetic resonance imaging. Invest Ophthalmol Vis Sci 45: 2722-2731.

32. Kim YM, Lim JO, Kim HK, Kim SY, Shin JP (2008) A novel design of oneside coated biodegradable intrascleral implant for the sustained release of triamcinolone acetonide. Eur J Pharm Biopharm 70: 179-186.

33. Gwon AE, Meadows D (1995) Subconjunctival implants for ocular drug delivery. Google patents.

34. Okabe J, Kimura H, Kunou N, Okabe K, Kato A, et al. (2003) Biodegradable Intrascleral Implant for Sustained Intraocular Delivery of Betamethasone Phosphate. Invest Ophthalmol Vis Sci 44: 740-744.

35. Manda P, Kushwaha AS, Kundu S, Shivakumar H, Jo SB, et al. (2016) Delivery of ziconotide to cerebrospinal fluid via intranasal pathway for the treatment of chronic pain. J Control Release 224: 69-76.

36. Michelson JB, Nozik RA (1979) Experimental endophthalmitis treated with an implantable osmotic minipump. Arch Ophthalmol 97: 1345-1346.

37. Fialho SL, Behar-Cohen F, Silva-Cunha A (2008) Dexamethasone-loaded poly(epsilon-caprolactone) intravitreal implants: a pilot study. Eur J Pharm Biopharm 68: 637-646.

38. Popescu C, Manda P, Juluri A, Janga KY, Cidda M, et al. (2015) Enhanced dissolution efficiency of zaleplon solid dispersions via modified ß-cyclodextrin molecular inclusion complexes. J Pharm Pharm Sci 1: 12-21.

39. Carcaboso AM, Chiappetta DA, Opezzo JA, Höcht C, Fandiño AC, et al. (2010) Episcleral implants for topotecan delivery to the posterior segment of the eye. Invest Ophthalmol Vis Sci 51: 2126-2134.

40. Choonara YE, Pillay V, Danckwerts MP, Carmichael TR, Meyer LC, et al (2010) In vivo evaluation of a biodegradable donut-shaped minitablet for prolonged posterior segment drug delivery in the rabbit eye model. J Pharm Sci 100: 1819-1832.

41. http://www.psivida.com/products-durasert.html

42. Manda P, Sammeta SM, Repka MA, Murthy SN (2012) lontophoresis across the proximal nail fold to target drugs to the nail matrix. J Pharm Sci 101: 2392-2397.

43. Dugel PU, Eliott D, Cantrill HL, Mahmoud T, Avery R, et al. (2009) I-VationTM TA: 24-month Clinical Results of the Phase I Safety and Preliminary Efficacy Study. Invest Ophthalmol Vis Sci 50: 4332.

44. Xu X, Al-Ghabeish M, Rahman Z, Krishnaiah YS, Yerlikaya F, et al. (2015) Formulation and process factors influencing product quality and in vitro performance of ophthalmic ointments. Int J Pharm 493: 412-425.

45. ClinicalTrials.gov, National Institutes of Health (2015) Safety and tolerability of NOVA63035 Corticosteroid? in patients with diabetic macular edema secondary to diabetic retinopathy. 